\title{
EFFECTS OF TOMATO HARVEST RESIDUE DERIVED BIOCHARS OBTAINED FROM DIFFERENT PYROLYSIS TEMPERATURE AND DURATION ON PLANT GROWTH AND NUTRIENT CONCENTRATIONS OF CORN
}

\author{
Ibrahim Erdal, Murat Memici, Ahmet Dogan, Cennet Yaylaci, Kamil Ekinci \\ University of Suleyman Demirel, Turkey \\ ibrahimerdal@sdu.edu.tr
}

\begin{abstract}
This study is aimed to investigate the effects of different biochars obtained from tomato harvest residues (THR) on the growth, nutrient concentrations and nutrient uptakes of corn plant. For this purpose, biochars were produced using THR, using a pyrolyser at temperature of $300,400,500,600$, and $700{ }^{\circ} \mathrm{C}$ with application duration of $40,80,120$, and 240 minutes. THR had $\mathrm{C}$ and $\mathrm{N}$ content of $22.12 \%$ and $2.00 \%$, respectively. Biochars obtained at five application temperatures and four application durations were applied with the rates of 10 and $40 \mathrm{t} \cdot \mathrm{ha}^{-1}$. The experiment was conducted in a greenhouse. After 2 months growth period, the plants were harvested above ground of the soil. Harvested plants were washed and dry weighted and mineral nutrient concentrations of the plants were determined. The results showed that low application rate $\left(10 \mathrm{t} \cdot \mathrm{ha}^{-1}\right)$ of biochar obtained at $300-600{ }^{\circ} \mathrm{C}$ and at longer duration increased the plant growth. However, there is no systematic effect of different biochar applications on nutrient concentrations of corn plant generally.
\end{abstract}

Keywords: biochar, nutrient, plant growth, pyrolysis, temperature, durations.

\section{Introduction}

The preservation and improvement of the physical, chemical and biological properties of the soil is very important in terms of conservation of sustainable soil fertility. In order to obtain abundant and quality products from plant production, it is possible that the environmental conditions, in which the plants are grown, are at optimum level in terms of the soil properties In addition to intensive and unconscious agricultural practices; the soil loses its efficiency over time, depending on the adverse effects of climate and environment. Inadequacy of soil organic matter, erosion, water scarcity, unconscious chemical input use, salinity, increase in evapotranspiration and natural or human-induced factors cause the yield potential of the soil to decrease gradually. For this reason, those engaged in agricultural science have searched for various ways to meet the increasing need for agricultural consumption. Researchers are trying to maintain the productivity of agricultural land on the one side and to increase production on the other. Although chemical fertilizers are the most effective way of improving crop production, they cause some negative effects on soil and environment due to unconscious practices. For this reason, applications that increase the efficiency of plants to utilize fertilizers or improve various properties of the soil have become increasingly important. One of these is biochar applications. Biochar can be obtained from any kinds of biomass material and gained from carbonization of the biomass. Biochar can increase soil fertility by incorporating carbon to the soil. Holding plant nutrients for a longer period of time within the root zone means higher fertilizer use efficiency. Leaching of plant nutrients below the root zone becomes both unavailable for plants and also leads to pollution by leaching to ground water and harms the ecosystem functions. Depending on the numerous factors indicated above, biochar increases the soil fertility, plant growth and yield [1-6]. Reduction in the quantity of agricultural waste is another advantage of biochar production. Tomato is one of the most important agricultural products in Turkey and quite amount of harvest residues exist at the production period. Burning of these residuals is a common application to be eliminated. This study offers a method of utilization of THR as biochar through slow pyrolysis of biomass.

This study is aimed to investigate the effects of different biochar obtained from tomato harvest residues (THR) on the growth, nutrient concentrations and nutrient uptakes of corn plant.

\section{Materials and methods}

This study was conducted as a pot experiment under greenhouse conditions under natural lighting. For this purpose, biochars were produced from THR using a pyrolyser at temperature of 300, 400, 500, 600 , and $700{ }^{\circ} \mathrm{C}$ with the application duration of 40, 80,120, and 240 minutes. THR had C and $\mathrm{N}$ content of $22.12 \%$ and $2.00 \%$, respectively. Biochars obtained at five application temperatures and four application durations were applied at the rates of 0 (control), 10 and $40 \mathrm{t} \cdot \mathrm{ha}^{-1}$. The experiment was arranged according to completely randomized design with three replicates including 180 pots in 
total. The pots were randomly rotated each day to a different position within the block for the duration of the trial.

Biochars were added to the soils in the $2.5 \mathrm{~kg}$ soil containing pots and mixed. At the same time, $300 \mathrm{mg} \cdot \mathrm{kg}^{-1} \mathrm{~N}$ (as ammonium nitrate), $200 \mathrm{mg} \cdot \mathrm{kg}^{-1} \mathrm{P}$ (as triple super phosphate) and $100 \mathrm{mg} \cdot \mathrm{kg}^{-1} \mathrm{P}$ (as potassium sulphate) were added to the soil as basal fertilization. As plant material, silage maize (KWS - Kolosseus) was used. The experiments were conducted for 2 months (June-July) and the plants were irrigated with tap water. The available nutrients in the soil were determined, phosphorus (P) extracted with sodium bicarbonate [7], potassium $(\mathrm{K})$, calcium $(\mathrm{Ca})$, and magnesium $(\mathrm{Mg})$ extracted with ammonium acetate [8] and manganese $(\mathrm{Mn})$, zinc $(\mathrm{Zn})$, iron $(\mathrm{Fe})$, and copper $(\mathrm{Cu})$ extracted with DTPA [9]. Phosphorus measurement was done using a spectrophotometer; others were measured with an atomic absorption spectrophotometer. Soil texture was determined using a hydrometer [10] and the calcium carbonate $\left(\mathrm{CaCO}_{3}\right)$ content was measured with a calcimeter [11]. Soil organic matter was determined based on Walkley and Black [12]. Soil $\mathrm{pH}$ was measured using $\mathrm{pH}$ meter in suspension of soil and water at the rates of $1 / 2.5$. Some physical and chemical properties of the experimental soils are given in Table 1.

Some physical and chemical characteristics of the experimental soils

Table 1

\begin{tabular}{|c|c|c|c|c|c|c|c|c|c|c|c|}
\hline \multirow{2}{*}{ Texture } & \multirow{2}{*}{ pH } & \multirow{2}{*}{$\underset{\%}{\mathrm{OM},}$} & \multirow{2}{*}{$\begin{array}{c}\mathrm{CaCO}_{3}, \\
\%\end{array}$} & \multicolumn{8}{|c|}{ Plant available nutrients, $\mathrm{mg} \cdot \mathrm{kg}^{-1}$} \\
\hline & & & & $\mathrm{P}$ & $\mathrm{K}$ & $\mathrm{Ca}$ & $\mathrm{Mg}$ & $\mathrm{Fe}$ & $\mathrm{Zn}$ & $\mathrm{Mn}$ & $\mathrm{Cu}$ \\
\hline Lo & 7.9 & 2.6 & 28 & 108 & 443 & 7500 & 180 & 10 & 1.1 & 7 & 2.5 \\
\hline
\end{tabular}

After 2 months growth period, the plants were harvested above ground of the soil. Harvested plants were washed, dried, weighted and prepared for the analyses. Total nitrogen was analysed according to the Kjeldahl method. Phosphorus concentrations of the samples were determined with a spectrophotometer (Shimadzu UV-1208) at $430 \mathrm{~nm}$ according to the vanadomolybdo phosphoric acid method. Potassium, $\mathrm{Ca}, \mathrm{Mg}, \mathrm{Fe}, \mathrm{Cu}, \mathrm{Zn}$, and $\mathrm{Mn}$ concentrations were determined using atomic absorption spectrophotometer [13]. All data concerning dry weight and nutrient concentrations were submitted for statistical analyses using MSTAT program for one-way analysis of variance applied to determine any significant difference at $0.05 \%$.

\section{Results and discussion}

Plant dry weight and macronutrient concentrations

Interaction of the pyrolysis temperature (PT), pyrolysis application duration (PAD) and application rates significantly affected the plant dry weight. Based on Table 2, it was clearly seen that although there were positive effects of some biochar treatments, there were negative effects of some others. The highest dry weight was obtained from the pot with the application rate of $10 \mathrm{t} \cdot \mathrm{ha}^{-1}$ of biochar at PT of $600{ }^{\circ} \mathrm{C}$ for 240 minutes. The lowest dry weight was obtained from the application rate of $40 \mathrm{t} \mathrm{ha}^{-1}$ of biochar at PT of $700{ }^{\circ} \mathrm{C}$ for 120 minutes. Although PT, PAD and application rates were not individually evaluated, the highest dry matter values was obtained from PAD of 240 minutes of each PT (except for $700{ }^{\circ} \mathrm{C}$ ). In general, the plant dry weight values obtained at higher application rates were lower than the control values in general, while the plant dry weight values in the lower application rates were higher than the control group (Table 2).

Interaction effect on the plant $\mathrm{N}$ concentration was found to be significant. The highest plant $\mathrm{N}$ concentration was obtained from the application rate of $40 \mathrm{t} \cdot \mathrm{ha}^{-1}$ of biochar at PT of $500{ }^{\circ} \mathrm{C}$ for 40 minutes. The nitrogen concentration values obtained from the other applications were either in the same group with the control or lower than the control group. Although interaction effects of factors such as the plant $\mathrm{Ca}$ concentrations were found to be significant, this effect was found to be negative in all subjects (Table 3).

The effect of biochar applications on the K concentration of the plant was generally similar to that obtained from the control group, whereas in some applications, more $\mathrm{K}$ values were determined than in the control group. There was no statistically significant effect of $\mathrm{Mg}$ on the plant $\mathrm{Mg}$ concentration (Table 4). 
Effects of biochar applications on dry weight

\begin{tabular}{|c|c|c|c|c|}
\hline \multirow{3}{*}{ PT, ${ }^{\circ} \mathrm{C}$} & \multirow{3}{*}{$\begin{array}{l}\text { PA, } \\
\text { min }\end{array}$} & \multicolumn{3}{|c|}{ Dry weight, $\mathrm{g}$} \\
\hline & & \multicolumn{3}{|c|}{ Application rates, $t \cdot h^{-1}$} \\
\hline & & 0 & 10 & 40 \\
\hline \multirow{4}{*}{300} & 40 & $0.60 \mathrm{~F}-\mathrm{K}^{*}$ & $0.60 \mathrm{~F}-\mathrm{K}$ & $0.77 \mathrm{D}-\mathrm{J}$ \\
\hline & 80 & $0.60 \mathrm{~F}-\mathrm{K}$ & $0.68 \mathrm{E}-\mathrm{J}$ & $0.71 \mathrm{D}-\mathrm{J}$ \\
\hline & 120 & $0.60 \mathrm{~F}-\mathrm{K}$ & $0.90 \mathrm{C}-\mathrm{F}$ & $0.66 \mathrm{~F}-\mathrm{J}$ \\
\hline & 240 & $0.60 \mathrm{~F}-\mathrm{K}$ & $1.1 \mathrm{~B} \mathrm{C}$ & $1.1 \mathrm{BC}$ \\
\hline \multirow{4}{*}{400} & 40 & $0.60 \mathrm{~F}-\mathrm{K}$ & $0.71 \mathrm{D}-\mathrm{J}$ & $0.45 \mathrm{JK}$ \\
\hline & 80 & $0.60 \mathrm{~F}-\mathrm{K}$ & $0.62 \mathrm{~F}-\mathrm{K}$ & $0.45 \mathrm{JK}$ \\
\hline & 120 & $0.60 \mathrm{~F}-\mathrm{K}$ & $0.68 \mathrm{E}-\mathrm{J}$ & $0.49 \mathrm{I}-\mathrm{K}$ \\
\hline & 240 & $0.60 \mathrm{~F}-\mathrm{K}$ & $1.40 \mathrm{AB}$ & $0.55 \mathrm{H}-\mathrm{K}$ \\
\hline \multirow{4}{*}{500} & 40 & $0.60 \mathrm{~F}-\mathrm{K}$ & $0.73 \mathrm{D}-\mathrm{J}$ & $0.52 \mathrm{I}-\mathrm{K}$ \\
\hline & 80 & $0.60 \mathrm{~F}-\mathrm{K}$ & $0.59 \mathrm{~F}-\mathrm{K}$ & $0.60 \mathrm{~F}-\mathrm{K}$ \\
\hline & 120 & $0.60 \mathrm{~F}-\mathrm{K}$ & $0.62 \mathrm{~F}-\mathrm{K}$ & $0.56 \mathrm{H}-\mathrm{K}$ \\
\hline & 240 & $0.60 \mathrm{~F}-\mathrm{K}$ & $1.10 \mathrm{BC}$ & $0.60 \mathrm{~F}-\mathrm{K}$ \\
\hline \multirow{4}{*}{600} & 40 & $0.60 \mathrm{~F}-\mathrm{K}$ & $0.87 \mathrm{C}-\mathrm{G}$ & $0.83 \mathrm{C}-\mathrm{H}$ \\
\hline & 80 & $0.60 \mathrm{~F}-\mathrm{K}$ & $0.81 \mathrm{D}-\mathrm{I}$ & $0.77 \mathrm{D}-\mathrm{J}$ \\
\hline & 120 & $0.60 \mathrm{~F}-\mathrm{K}$ & $0.74 \mathrm{D}-\mathrm{J}$ & $0.71 \mathrm{D}-\mathrm{J}$ \\
\hline & 240 & $0.60 \mathrm{~F}-\mathrm{K}$ & $1.60 \mathrm{~A}$ & 0.66 F-J \\
\hline \multirow{4}{*}{700} & 40 & $0.60 \mathrm{~F}-\mathrm{K}$ & 0.75 D-J & $0.55 \mathrm{H}-\mathrm{K}$ \\
\hline & 80 & $0.60 \mathrm{~F}-\mathrm{K}$ & 0.65 E-J & $0.45 \mathrm{JK}$ \\
\hline & 120 & $0.60 \mathrm{~F}-\mathrm{K}$ & $0.84 \mathrm{~F}-\mathrm{K}$ & $0.33 \mathrm{~K}$ \\
\hline & 240 & $0.60 \mathrm{~F}-\mathrm{K}$ & 0.67 F-J & $0.49 \mathrm{I}-\mathrm{K}$ \\
\hline
\end{tabular}

*: There is no statistical difference between the values that share the same letter $(P<0.05)$

Table 3

Effects of biochar applications on plant $\mathbf{N}$ and Ca concentrations

\begin{tabular}{|c|c|c|c|c|c|c|c|}
\hline \multirow{3}{*}{ PT, ${ }^{\circ} \mathrm{C}$} & \multirow{3}{*}{$\begin{array}{l}\text { PAD, } \\
\text { min }\end{array}$} & \multicolumn{3}{|c|}{$\mathrm{N}, \%$} & \multirow{2}{*}{\multicolumn{3}{|c|}{$\begin{array}{c}\text { Ca, \% } \\
\text { Application rates, } \mathbf{t} \cdot \mathbf{h a}^{-1}\end{array}$}} \\
\hline & & Appl & ation rate & $t \cdot h a^{-1}$ & & & \\
\hline & & 0 & 10 & 40 & 0 & 10 & 40 \\
\hline \multirow{4}{*}{300} & 40 & $3.1 \mathrm{AB}^{*}$ & $2.0 \mathrm{C}-\mathrm{H}$ & $3.2 \mathrm{AB}$ & $0.31 \mathrm{~A}-\mathrm{E}$ & $0.27 \mathrm{~A}-\mathrm{D}$ & $0.25 \mathrm{~A}-\mathrm{D}$ \\
\hline & 80 & $3.1 \mathrm{AB}$ & $2.7 \mathrm{C}-\mathrm{H}$ & $3.0 \mathrm{~A}-\mathrm{C}$ & $0.31 \mathrm{~A}-\mathrm{E}$ & $0.21 \mathrm{~B}-\mathrm{D}$ & 0.24 B-D \\
\hline & 120 & $3.1 \mathrm{AB}$ & $2.0 \mathrm{C}-\mathrm{H}$ & $3.3 \mathrm{~A}-\mathrm{B}$ & $0.31 \mathrm{~A}-\mathrm{E}$ & $0.21 \mathrm{~B}-\mathrm{D}$ & $0.32 \mathrm{~A}-\mathrm{D}$ \\
\hline & 240 & $3.1 \mathrm{AB}$ & $2.0 \mathrm{C}-\mathrm{H}$ & $2.3 \mathrm{~B}-\mathrm{H}$ & $0.31 \mathrm{~A}-\mathrm{E}$ & 0.19 B-D & $0.24 \mathrm{~B}-\mathrm{D}$ \\
\hline \multirow{4}{*}{400} & 40 & $3.1 \mathrm{AB}$ & $2.4 \mathrm{~B}-\mathrm{H}$ & $2.3 \mathrm{~B}-\mathrm{H}$ & $0.31 \mathrm{~A}-\mathrm{E}$ & 0.22 B-D & $0.24 \mathrm{~B}-\mathrm{D}$ \\
\hline & 80 & $3.1 \mathrm{AB}$ & $2.0 \mathrm{C}-\mathrm{H}$ & $2.7 \mathrm{~A}-\mathrm{G}$ & $0.31 \mathrm{~A}-\mathrm{E}$ & 0.22 B-D & $0.21 \mathrm{~B}-\mathrm{D}$ \\
\hline & 120 & $3.1 \mathrm{AB}$ & $2.3 \mathrm{~B}-\mathrm{H}$ & $2.0 \mathrm{C}-\mathrm{H}$ & $0.31 \mathrm{~A}-\mathrm{E}$ & 0.24 B-D & $0.18 \mathrm{CD}$ \\
\hline & 240 & $3.1 \mathrm{AB}$ & $1.7 \mathrm{G} \mathrm{H}$ & $2.3 \mathrm{~B}-\mathrm{H}$ & $0.31 \mathrm{~A}-\mathrm{E}$ & $0.19 \mathrm{CD}$ & $0.24 \mathrm{~B}-\mathrm{D}$ \\
\hline \multirow{4}{*}{500} & 40 & $3.1 \mathrm{AB}$ & $1.9 \mathrm{D}-\mathrm{H}$ & $3.7 \mathrm{~A}$ & $0.31 \mathrm{~A}-\mathrm{E}$ & $0.21 \mathrm{~B}-\mathrm{D}$ & 0.29 A-D \\
\hline & 80 & $3.1 \mathrm{AB}$ & $1.97 \mathrm{D}-\mathrm{H}$ & 2.9 A-D & $0.31 \mathrm{~A}-\mathrm{E}$ & $0.26 \mathrm{~A}-\mathrm{D}$ & $0.19 \mathrm{~B}-\mathrm{D}$ \\
\hline & 120 & $3.1 \mathrm{AB}$ & $2.4 \mathrm{~B}-\mathrm{H}$ & $2.7 \mathrm{~A}-\mathrm{G}$ & $0.31 \mathrm{~A}-\mathrm{E}$ & 0.22 B-D & $0.27 \mathrm{~A}-\mathrm{D}$ \\
\hline & 240 & $3.1 \mathrm{AB}$ & $1.9 \mathrm{D}-\mathrm{H}$ & 2.8 A-E & $0.31 \mathrm{~A}-\mathrm{E}$ & $0.21 \mathrm{~B}-\mathrm{D}$ & $0.26 \mathrm{~A}-\mathrm{D}$ \\
\hline \multirow{4}{*}{600} & 40 & $3.1 \mathrm{AB}$ & $2.1 \mathrm{C}-\mathrm{H}$ & $2.0 \mathrm{C}-\mathrm{H}$ & $0.31 \mathrm{~A}-\mathrm{E}$ & $0.18 \mathrm{CD}$ & 0.29 A-D \\
\hline & 80 & $3.1 \mathrm{AB}$ & $1.6 \mathrm{H}$ & $2.2 \mathrm{C}-\mathrm{H}$ & $0.31 \mathrm{~A}-\mathrm{E}$ & $0.18 \mathrm{CD}$ & $0.31 \mathrm{~A}-\mathrm{E}$ \\
\hline & 120 & $3.1 \mathrm{AB}$ & $2.0 \mathrm{C}-\mathrm{H}$ & $2.2 \mathrm{C}-\mathrm{H}$ & $0.31 \mathrm{~A}-\mathrm{E}$ & $0.18 \mathrm{CD}$ & $0.27 \mathrm{~A}-\mathrm{D}$ \\
\hline & 240 & $3.1 \mathrm{AB}$ & $1.6 \mathrm{~B}-\mathrm{H}$ & $2.1 \mathrm{C}-\mathrm{H}$ & $0.31 \mathrm{~A}-\mathrm{E}$ & $0.16 \mathrm{D}$ & $0.27 \mathrm{~A}-\mathrm{D}$ \\
\hline \multirow{4}{*}{700} & 40 & $3.1 \mathrm{AB}$ & $1.7 \mathrm{~F}-\mathrm{H}$ & $2.1 \mathrm{C}-\mathrm{H}$ & $0.31 \mathrm{~A}-\mathrm{E}$ & 0.20 B-D & $0.27 \mathrm{~A}-\mathrm{D}$ \\
\hline & 80 & $3.1 \mathrm{AB}$ & $1.7 \mathrm{~F}-\mathrm{H}$ & $2.3 \mathrm{~B}-\mathrm{H}$ & $0.31 \mathrm{~A}-\mathrm{E}$ & 0.23 B-D & $0.24 \mathrm{~B}-\mathrm{D}$ \\
\hline & 120 & $3.1 \mathrm{AB}$ & $1.8 \mathrm{E}-\mathrm{H}$ & $2.5 \mathrm{~B}-\mathrm{H}$ & $0.31 \mathrm{~A}-\mathrm{E}$ & 0.22 B-D & $0.31 \mathrm{~A}-\mathrm{C}$ \\
\hline & 240 & $3.1 \mathrm{AB}$ & $2.4 \mathrm{~B}-\mathrm{H}$ & $2.5 \mathrm{~B}-\mathrm{H}$ & $0.31 \mathrm{~A}-\mathrm{E}$ & $0.25 \mathrm{~A}-\mathrm{D}$ & $0.28 \mathrm{~A}-\mathrm{D}$ \\
\hline
\end{tabular}

*: There is no statistical difference between the values that share the same letter $(P<0.05)$ 
Effects of biochar applications on plant $\mathrm{K}$ and Mg concentrations

Table 4

\begin{tabular}{|c|c|c|c|c|c|c|c|}
\hline \multirow{3}{*}{ PT, ${ }^{\circ} \mathrm{C}$} & \multirow{3}{*}{$\begin{array}{c}\text { PAD, } \\
\text { min }\end{array}$} & \multicolumn{3}{|c|}{$\mathrm{K}, \%$} & \multicolumn{3}{|c|}{ Mg, \% } \\
\hline & & \multicolumn{3}{|c|}{ Application rates, $\mathrm{t} \cdot \mathrm{ha}^{-1}$} & \multicolumn{3}{|c|}{ Application rates, $\mathbf{t} \cdot \mathbf{h a}^{-1}$} \\
\hline & & 0 & 10 & 40 & 0 & 10 & 40 \\
\hline \multirow{4}{*}{300} & 40 & 3.99 B-E* & $3.7 \mathrm{~B}-\mathrm{E}$ & $4.6 \mathrm{~A}-\mathrm{D}$ & 0.52 & 0.46 & 0.48 \\
\hline & 80 & $3.99 \mathrm{~B}-\mathrm{E}$ & $4.1 \mathrm{~A}-\mathrm{E}$ & $3.4 \mathrm{D} \mathrm{E}$ & 0.52 & 0.46 & 0.49 \\
\hline & 120 & $3.99 \mathrm{~B}-\mathrm{E}$ & $3.7 \mathrm{~B}-\mathrm{E}$ & $4.4 \mathrm{~A}-\mathrm{E}$ & 0.52 & 0.46 & 0.54 \\
\hline & 240 & 3.99 B-E & 3.9 B-E & 4.4 A-E & 0.52 & 0.45 & 0.48 \\
\hline \multirow{4}{*}{400} & 40 & $3.99 \mathrm{~B}-\mathrm{E}$ & $4.5 \mathrm{~A}-\mathrm{D}$ & $4.1 \mathrm{~A}-\mathrm{E}$ & 0.52 & 0.48 & 0.48 \\
\hline & 80 & 3.99 B-E & $4.7 \mathrm{~A}-\mathrm{D}$ & $4.98 \mathrm{~A}-\mathrm{D}$ & 0.52 & 0.50 & 0.45 \\
\hline & 120 & $3.99 \mathrm{~B}-\mathrm{E}$ & $4.4 \mathrm{~A}-\mathrm{E}$ & $4.1 \mathrm{~A}-\mathrm{E}$ & 0.52 & 0.49 & 0.45 \\
\hline & 240 & 3.99 B-E & $4.0 \mathrm{~A}-\mathrm{E}$ & $4.4 \mathrm{~A}-\mathrm{E}$ & 0.52 & 0.40 & 0.48 \\
\hline \multirow{4}{*}{500} & 40 & $3.99 \mathrm{~B}-\mathrm{E}$ & $4.8 \mathrm{~A}-\mathrm{D}$ & 4.8 A-D & 0.52 & 0.49 & 0.47 \\
\hline & 80 & $3.99 \mathrm{~B}-\mathrm{E}$ & $3.8 \mathrm{~B}-\mathrm{E}$ & 4.7 A-D & 0.52 & 0.46 & 0.46 \\
\hline & 120 & $3.99 \mathrm{~B}-\mathrm{E}$ & $4.1 \mathrm{~A}-\mathrm{E}$ & $5.5 \mathrm{~A}-\mathrm{C}$ & 0.52 & 0.43 & 0.41 \\
\hline & 240 & 3.99 B-E & $3.9 \mathrm{~B}-\mathrm{E}$ & $4.1 \mathrm{~A}-\mathrm{E}$ & 0.52 & 0.41 & 0.46 \\
\hline \multirow{4}{*}{600} & 40 & $3.99 \mathrm{~B}-\mathrm{E}$ & $4.1 \mathrm{~A}-\mathrm{E}$ & $4.6 \mathrm{~A}-\mathrm{D}$ & 0.52 & 0.40 & 0.54 \\
\hline & 80 & 3.99 B-E & $4.1 \mathrm{~A}-\mathrm{E}$ & $5.5 \mathrm{~A}-\mathrm{C}$ & 0.52 & 0.49 & 0.56 \\
\hline & 120 & $3.99 \mathrm{~B}-\mathrm{E}$ & $4.0 \mathrm{~A}-\mathrm{E}$ & $5.3 \mathrm{~A}-\mathrm{D}$ & 0.52 & 0.47 & 0.52 \\
\hline & 240 & $3.99 \mathrm{~B}-\mathrm{E}$ & $4.2 \mathrm{~A}-\mathrm{E}$ & $5.6 \mathrm{~A}-\mathrm{B}$ & 0.52 & 0.48 & 0.59 \\
\hline \multirow{4}{*}{700} & 40 & 3.99 B-E & $3.6 \mathrm{~B}-\mathrm{E}$ & $5.9 \mathrm{~A}$ & 0.52 & 0.48 & 0.57 \\
\hline & 80 & $3.99 \mathrm{~B}-\mathrm{E}$ & $3.8 \mathrm{~B}-\mathrm{E}$ & $5.6 \mathrm{~A} \mathrm{~B}$ & 0.52 & 0.50 & 0.53 \\
\hline & 120 & 3.99 B-E & $3.8 \mathrm{~B}-\mathrm{E}$ & $4.3 \mathrm{~A}-\mathrm{E}$ & 0.52 & 0.46 & 0.48 \\
\hline & 240 & 3.99 B-E & $4.1 \mathrm{~A}-\mathrm{E}$ & $46 \mathrm{~A}-\mathrm{D}$ & 0.52 & 0.51 & 0.49 \\
\hline
\end{tabular}

*: There is no statistical difference between the values that share the same $(P<0.05)$

\section{Micronutrient concentrations}

The effect of biochar applications on the $\mathrm{Cu}$ concentration of corn was found to be negative. It is noteworthy that applications at the plant Mn concentrations had different effects. It was observed that the plant $\mathrm{Mn}$ concentrations were affected by the applications. While some applications were adversely affected, some applications were found to be at the same statistical group as the values obtained from the control group, and the values obtained from some applications were found to be higher than those of the control (Table 5). Similar cases were observed in the plant Fe and $\mathrm{Zn}$ concentrations (Table 6).

Table 5

Effects of biochar applications on plant $\mathrm{Cu}$ and Mn concentrations

\begin{tabular}{|c|c|c|c|c|c|c|c|}
\hline \multirow{3}{*}{ PT, ${ }^{\circ} \mathrm{C}$} & \multirow{3}{*}{$\begin{array}{l}\text { PAD, } \\
\text { min }\end{array}$} & \multicolumn{3}{|c|}{$\mathrm{Cu}, \mathrm{mg} \cdot \mathrm{kg}^{-1}$} & \multicolumn{3}{|c|}{ Mn, $\mathbf{m g} \cdot \mathbf{k g}^{-1}$} \\
\hline & & \multicolumn{3}{|c|}{ Application rates, $\mathrm{t} \cdot \mathrm{ha}^{-1}$} & \multicolumn{3}{|c|}{ Application rates, $t \cdot h^{-1}$} \\
\hline & & 0 & 10 & 40 & 0 & 10 & 40 \\
\hline \multirow{4}{*}{300} & 40 & $49.8 \mathrm{~A}$ & $19.5 \mathrm{BC}$ & $15.9 \mathrm{BC}$ & $104 \mathrm{D}-\mathrm{G}$ & 83 G-N & 88 F-N \\
\hline & 80 & $49.8 \mathrm{~A}$ & $13.0 \mathrm{C}$ & $17.9 \mathrm{BC}$ & $104 \mathrm{D}-\mathrm{G}$ & $104 \mathrm{C}-\mathrm{K}$ & $84 \mathrm{O}$ \\
\hline & 120 & $49.8 \mathrm{~A}$ & $13.6 \mathrm{BC}$ & $16.4 \mathrm{BC}$ & $104 \mathrm{D}-\mathrm{G}$ & $103 \mathrm{C}-\mathrm{L}$ & $74 \mathrm{M} \mathrm{N}$ \\
\hline & 240 & $49.8 \mathrm{~A}$ & $11.7 \mathrm{C}$ & $16.0 \mathrm{BC}$ & $104 \mathrm{D}-\mathrm{G}$ & $79 \mathrm{I}-\mathrm{N}$ & $82 \mathrm{H}-\mathrm{N}$ \\
\hline \multirow{4}{*}{400} & 40 & $49.8 \mathrm{~A}$ & $14.7 \mathrm{BC}$ & $16.0 \mathrm{BC}$ & $104 \mathrm{D}-\mathrm{G}$ & $76 \mathrm{~L}-\mathrm{N}$ & $84 \mathrm{G}-\mathrm{N}$ \\
\hline & 80 & $49.8 \mathrm{~A}$ & $14 \mathrm{BC}$ & $15.0 \mathrm{BC}$ & $104 \mathrm{D}-\mathrm{G}$ & $92 \mathrm{E}-\mathrm{N}$ & $68 \mathrm{M}-\mathrm{O}$ \\
\hline & 120 & $49.8 \mathrm{~A}$ & $17 \mathrm{BC}$ & $25.3 \mathrm{BC}$ & $104 \mathrm{D}-\mathrm{G}$ & $91 \mathrm{E}-\mathrm{N}$ & $118 \mathrm{~A}-\mathrm{E}$ \\
\hline & 240 & $49.8 \mathrm{~A}$ & $14 \mathrm{BC}$ & $16.0 \mathrm{BC}$ & $104 \mathrm{D}-\mathrm{G}$ & $79 \mathrm{~J}-\mathrm{N}$ & $82 \mathrm{H}-\mathrm{N}$ \\
\hline \multirow{4}{*}{500} & 40 & $49.8 \mathrm{~A}$ & $24.2 \mathrm{BC}$ & $18.0 \mathrm{BC}$ & $104 \mathrm{D}-\mathrm{G}$ & $110 \mathrm{~A}-\mathrm{G}$ & $68 \mathrm{M}-\mathrm{O}$ \\
\hline & 80 & $49.8 \mathrm{~A}$ & $11.6 \mathrm{C}$ & $14.7 \mathrm{BC}$ & $104 \mathrm{D}-\mathrm{G}$ & 94 E-M & $66 \mathrm{~N} \mathrm{O}$ \\
\hline & 120 & $49.8 \mathrm{~A}$ & $14.8 \mathrm{BC}$ & $15.3 \mathrm{BC}$ & $104 \mathrm{D}-\mathrm{G}$ & $107 \mathrm{~A}-\mathrm{I}$ & 91 E-N \\
\hline & 240 & $49.8 \mathrm{~A}$ & $20.0 \mathrm{BC}$ & $15.0 \mathrm{BC}$ & $104 \mathrm{D}-\mathrm{G}$ & $103 \mathrm{C}-\mathrm{L}$ & $105 \mathrm{~B}-\mathrm{K}$ \\
\hline
\end{tabular}


Table 5 (continued)

\begin{tabular}{|c|c|c|c|c|c|c|c|}
\hline \multirow{3}{*}{ PT, ${ }^{\circ} \mathrm{C}$} & \multirow{3}{*}{$\begin{array}{l}\text { PAD, } \\
\text { min }\end{array}$} & \multicolumn{3}{|c|}{$\mathrm{Cu}, \mathrm{mg} \cdot \mathrm{kg}^{-1}$} & \multicolumn{3}{|c|}{ Mn, $\mathrm{mg} \cdot \mathrm{kg}^{-1}$} \\
\hline & & \multicolumn{3}{|c|}{ Application rates, $t \cdot h^{-1}$} & \multicolumn{3}{|c|}{ Application rates, $\mathbf{t} \cdot \mathrm{ha}^{-1}$} \\
\hline & & 0 & 10 & 40 & 0 & 10 & 40 \\
\hline \multirow{4}{*}{600} & 40 & $49.8 \mathrm{~A}$ & $12.0 \mathrm{C}$ & $25.1 \mathrm{BC}$ & $104 \mathrm{D}-\mathrm{G}$ & 94 E-M & $104 \mathrm{C}-\mathrm{K}$ \\
\hline & 80 & $49.8 \mathrm{~A}$ & $11.9 \mathrm{C}$ & $25.6 \mathrm{BC}$ & $104 \mathrm{D}-\mathrm{G}$ & $95 \mathrm{E}-\mathrm{M}$ & $113 \mathrm{~A}-\mathrm{F}$ \\
\hline & 120 & $49.8 \mathrm{~A}$ & $25.3 \mathrm{BC}$ & $16.5 \mathrm{BC}$ & $104 \mathrm{D}-\mathrm{G}$ & $118 \mathrm{~A}-\mathrm{E}$ & $105 \mathrm{~B}-\mathrm{K}$ \\
\hline & 240 & $49.8 \mathrm{~A}$ & $15.0 \mathrm{BC}$ & $14.6 \mathrm{BC}$ & 104 D-G & $88 \mathrm{~F}-\mathrm{N}$ & $108 \mathrm{~A}-\mathrm{H}$ \\
\hline \multirow{4}{*}{700} & 40 & $49.8 \mathrm{~A}$ & $15.2 \mathrm{BC}$ & $24.0 \mathrm{~B}-\mathrm{C}$ & 104 D-G & $105 \mathrm{~B}-\mathrm{K}$ & $134 \mathrm{~A}$ \\
\hline & 80 & $49.8 \mathrm{~A}$ & $13.2 \mathrm{BC}$ & $18.9 \mathrm{BC}$ & $104 \mathrm{D}-\mathrm{G}$ & $128 \mathrm{~A}-\mathrm{D}$ & $132 \mathrm{~A} \mathrm{~B}$ \\
\hline & 120 & $49.8 \mathrm{~A}$ & $15.5 \mathrm{BC}$ & $15.4 \mathrm{BC}$ & $104 \mathrm{D}-\mathrm{G}$ & $117 \mathrm{~A}-\mathrm{E}$ & $128 \mathrm{~A}-\mathrm{C}$ \\
\hline & 240 & $49.8 \mathrm{~A}$ & $13.8 \mathrm{BC}$ & $26.7 \mathrm{BC}$ & $104 \mathrm{D}-\mathrm{G}$ & $111 \mathrm{~A}-\mathrm{F}$ & $115 \mathrm{~A}-\mathrm{F}$ \\
\hline
\end{tabular}

*: There is no statistical difference between the values that share the same $(P<0.05)$

Generally, the application rate of $10 \mathrm{t} \cdot \mathrm{ha}^{-1}$ of biochars obtained from up to PT of $600{ }^{\circ} \mathrm{C}$ for 240 minutes positively influenced the plant dry weights. Increasing the application rates led to a general decrease in the plant dry weight [14]. Various studies revealed different results for the effect of biochar on plant development. In a study conducted by Jones et al., [15] for 3 years, it was determined that there was no effect on the total biomass of biochar on corn growth and this was associated with an adequate supply of inorganic fertilizers. The increase in the plant dry weight was probably due to the soil nutrient supply capacity of biochar $[3 ; 6 ; 16]$, cation exchange capacity [3; 17], water retention capacity [3], microbial properties of soil [18; 19], soil acidity and electrical conductivity $[1 ; 20]$.

Table 6

Effects of biochar applications on plant $\mathrm{Fe}$ and $\mathrm{Zn}$ concentrations

\begin{tabular}{|c|c|c|c|c|c|c|c|}
\hline \multirow{3}{*}{ PT, ${ }^{\circ} \mathrm{C}$} & \multirow{3}{*}{$\begin{array}{c}\text { PAD, } \\
\text { min }\end{array}$} & \multicolumn{3}{|c|}{ Fe, $\mathrm{mg} \mathbf{k g}^{-1}$} & \multicolumn{3}{|c|}{$\mathrm{Zn}, \mathbf{m g} \cdot \mathrm{kg}^{-1}$} \\
\hline & & \multicolumn{3}{|c|}{ Application rates, $\mathbf{t} \cdot \mathbf{h a}^{-1}$} & \multicolumn{3}{|c|}{ Application rates, $\mathbf{t} \cdot \mathbf{h a}^{-1}$} \\
\hline & & 0 & 10 & 40 & 0 & 10 & 40 \\
\hline \multirow{4}{*}{300} & 40 & $202 \mathrm{C}-\mathrm{E}^{*}$ & $367 \mathrm{~B}$ & $189 \mathrm{C}-\mathrm{F}$ & $72 \mathrm{AB}$ & $33 \mathrm{E}-\mathrm{H}$ & $35 \mathrm{E}-\mathrm{H}$ \\
\hline & 80 & $202 \mathrm{C}-\mathrm{E}$ & $189 \mathrm{C}-\mathrm{F}$ & $176 \mathrm{C}-\mathrm{F}$ & $72 \mathrm{AB}$ & $31 \mathrm{~F}-\mathrm{H}$ & $33 \mathrm{E}-\mathrm{H}$ \\
\hline & 120 & 202 C-E & $132 \mathrm{EF}$ & 312 B C & $72 \mathrm{AB}$ & $25.3 \mathrm{H}$ & $39 \mathrm{D}-\mathrm{H}$ \\
\hline & 240 & 202 C-E & $173 \mathrm{C}-\mathrm{F}$ & $150 \mathrm{EF}$ & $72 \mathrm{AB}$ & $24.2 \mathrm{H}$ & $54 \mathrm{~B}-\mathrm{F}$ \\
\hline \multirow{4}{*}{400} & 40 & $202 \mathrm{C}-\mathrm{E}$ & $119 \mathrm{EF}$ & $166 \mathrm{C}-\mathrm{F}$ & $72 \mathrm{AB}$ & $37 \mathrm{D}$ & $53 \mathrm{~B}-\mathrm{G}$ \\
\hline & 80 & $202 \mathrm{C}-\mathrm{E}$ & $158 \mathrm{D}-\mathrm{F}$ & $139 \mathrm{EF}$ & $72 \mathrm{AB}$ & $21.0 \mathrm{H}$ & $36 \mathrm{D}-\mathrm{H}$ \\
\hline & 120 & $202 \mathrm{C}-\mathrm{E}$ & $176 \mathrm{C}-\mathrm{F}$ & $165 \mathrm{C}-\mathrm{F}$ & $72 \mathrm{AB}$ & $26.3 \mathrm{G} \mathrm{H}$ & $30 \mathrm{~F}-\mathrm{H}$ \\
\hline & 240 & $202 \mathrm{C}-\mathrm{E}$ & $108 \mathrm{~F}$ & $150 \mathrm{EF}$ & $72 \mathrm{AB}$ & $18.0 \mathrm{H}$ & $54 \mathrm{~B}-\mathrm{F}$ \\
\hline \multirow{4}{*}{500} & 40 & $202 \mathrm{C}-\mathrm{E}$ & $246 \mathrm{~B}-\mathrm{F}$ & $173 \mathrm{C}-\mathrm{F}$ & $72 \mathrm{AB}$ & $37 \mathrm{D}-\mathrm{H}$ & $44 \mathrm{C}-\mathrm{H}$ \\
\hline & 80 & $202 \mathrm{C}-\mathrm{E}$ & $179 \mathrm{C}-\mathrm{F}$ & $208 \mathrm{C}-\mathrm{F}$ & $72 \mathrm{AB}$ & $22.7 \mathrm{H}$ & $35 \mathrm{E}-\mathrm{H}$ \\
\hline & 120 & $202 \mathrm{C}-\mathrm{E}$ & $187 \mathrm{C}-\mathrm{F}$ & $261 \mathrm{~B}-\mathrm{E}$ & $72 \mathrm{AB}$ & $29.5 \mathrm{~F}-\mathrm{H}$ & $41 \mathrm{D}-\mathrm{H}$ \\
\hline & 240 & 202 C-E & $132 \mathrm{EF}$ & $124 \mathrm{EF}$ & $72 \mathrm{AB}$ & $31 \mathrm{~F}-\mathrm{H}$ & $54 \mathrm{~B}-\mathrm{F}$ \\
\hline \multirow{4}{*}{600} & 40 & $202 \mathrm{C}-\mathrm{E}$ & $172 \mathrm{C}-\mathrm{F}$ & $226 \mathrm{~B}-\mathrm{F}$ & $72 \mathrm{AB}$ & $22.0 \mathrm{H}$ & $62 \mathrm{~B}-\mathrm{D}$ \\
\hline & 80 & $202 \mathrm{C}-\mathrm{E}$ & $182 \mathrm{C}-\mathrm{F}$ & $357 \mathrm{~B}$ & $72 \mathrm{AB}$ & $18.7 \mathrm{H}$ & $44 \mathrm{C}-\mathrm{H}$ \\
\hline & 120 & $202 \mathrm{C}-\mathrm{E}$ & $165 \mathrm{C}-\mathrm{F}$ & $183 \mathrm{C}-\mathrm{F}$ & $72 \mathrm{AB}$ & $30 \mathrm{~F}-\mathrm{H}$ & $39 \mathrm{D}-\mathrm{H}$ \\
\hline & 240 & $202 \mathrm{C}-\mathrm{E}$ & $150 \mathrm{EF}$ & $172 \mathrm{C}-\mathrm{F}$ & $72 \mathrm{AB}$ & $35 \mathrm{E}-\mathrm{H}$ & $90 \mathrm{~A}$ \\
\hline \multirow{4}{*}{700} & 40 & $202 \mathrm{C}-\mathrm{E}$ & $180 \mathrm{C}-\mathrm{F}$ & 304 B-D & $72 \mathrm{AB}$ & $24.0 \mathrm{H}$ & $59 \mathrm{~B}-\mathrm{E}$ \\
\hline & 80 & $202 \mathrm{C}-\mathrm{E}$ & $182 \mathrm{C}-\mathrm{F}$ & $348 \mathrm{~B}$ & $72 \mathrm{AB}$ & $27.8 \mathrm{~F}-\mathrm{H}$ & $75 \mathrm{~A} \mathrm{~B}$ \\
\hline & 120 & 202 C-E & 249 B-F & $240 \mathrm{~B}-\mathrm{F}$ & $72 \mathrm{AB}$ & $29.0 \mathrm{~F}-\mathrm{H}$ & $39 \mathrm{D}-\mathrm{H}$ \\
\hline & 240 & 202 C-E & $148 \mathrm{C}-\mathrm{F}$ & 300 B-D & $72 \mathrm{AB}$ & $29.3 \mathrm{~F}-\mathrm{H}$ & $70 \mathrm{~A}-\mathrm{C}$ \\
\hline
\end{tabular}

*: There is no statistical difference between the values that share the same $(P<0.05)$

The effect of biochar applications on the nutrient concentrations of corn plants was rather variable. In all of the applications, a decrease in other nutrient concentrations of corn except potassium 
was recorded, but some increases were also observed. However, no systematic change was observed in these increases or decreases. It could be stated that it was possible to link some of the reductions in nutrient concentrations to plant development $[21 ; 22]$. In various studies, it was expressed that biochar reduces the availability of plant nutrients by absorbing [23; 24]. Although some applications cause reductions in plant nutrient concentrations, the determined values generally remained within the standard ranges considered sufficient for corn [13].

\section{Conclusions}

In the conclusion, the low application rate $\left(10 \mathrm{t} \cdot \mathrm{ha}^{-1}\right)$ of biochar obtained at $300-600{ }^{\circ} \mathrm{C}$ and at longer duration increased the plant growth. However, there is no systematic effect of different biochar applications on nutrient concentrations of corn plant generally.

\section{References}

[1] Asai H., Samson B.K., Stephan H.M. etc. Biochar amendment techniques for upland rice production in Northern Laos: 1. Soil physical properties, leaf SPAD and grain yield. Field Crop Res. 111, 2009, pp. 81-84

[2] Atkinson C.J., Fitzgerald J.D., Hipps N.A. Potential mechanisms for achieving agricultural benefits from biochar application to temperate soils: a review. Plant Soil 337, 2010, pp. 1-18.

[3] Glaser B., Lehmann J., Zech W. Ameliorating physical and chemical properties of highly weathered soils in the tropics with charcoal-a review. Biol. Fert. Soils 35, 2002, pp. 219-230.

[4] Lehmann J., Rondon M. Bio-char soil management on highly weathered soils in the humid tropics. In: Biol. Approaches to Sustain. Soil Syst. CRC Press, Boca Raton FL, 2006, pp. 517-530

[5] Lehmann J., Steiner C. Amazonian Dark Earths: Wim Sombroek's Vision. Springer Netherlands, Dordrecht. 2009.

[6] Major J., Lehmann J., Rondon M. etc. Fate of soil-applied black carbon: downward migration, leaching and soil respiration. Glob. Chang. Biol. 16, 2010, 1366-1379.

[7] Olsen S. R., Cole V., Watanabe F. S., Dean L. A. Estimation of available phosphorus in soils by extraction with sodium bicarbonate. Vol. 939. Washington D.C.: U.S. Department of Agriculture. 1954.

[8] Jackson M. L. 1967. Soil chemical analysis. New Delhi: Prentice Hall of India Private Limited.

[9] Lindsay W. L., Norvell W. A. Development of a DTPA soil test for zinc, iron, manganese, and copper1. Soil science society of America journal, 42(3), 1978), pp. 421-428.

[10] Bouyoucos G. L. A recalibration of the hydrometer for making mechanical analysis of soil. Agronomy Journal, 43, 1951, pp. 434-437.

[11] Allison L. E., Moodie C.D. Carbonate. In Methods of soil analysis, Part 2, ed. C. A. Black et al., Agronomy 9: 1965, pp.1379-1400.

[12] Walkley A., Black I A. A critical examination of a rapid method for determining organic carbon in soil-effect of variation in digestions and of inorganic soil constituents. Soil Sci. 63, 1947, pp. 251-263.

[13] Jones Jr J. B., Wolf B., Mills H. A. Plant analysis handbook. A practical sampling, preparation, analysis, and interpretation guide. Micro-Macro Publishing, Inc.1991.

[14] Gaskin J. W., Speir R. A., Harris K., Das K. C. etc. Effect of peanut hull and pine chip biochar on soil nutrients, corn nutrient status, and yield. Agronomy Journal, 102(2), 2010, pp. 623-633.

[15] Jones D. L., Rousk J., Edwards-Jones G. etc. Biochar-mediated changes in soil quality and plant growth in a three year field trial. Soil Biology and Biochemistry, 45, 2012, pp. 113-124.

[16] Lehmann J., Pereira da Silva J., Steiner C. etc. Nutrient availability and leaching in an archaeological Anthrosol and a Ferralsol of the Central Amazon basin: fertilizer, manure and charcoal amendments. Plant Soil 249, 2003, pp. 343-357.

[17] Topoliantz S., Ponge J. F., Arrouays D. Etc. Effect of organic manure and the endogeic earthworm Pontoscolex corethrurus (Oligochaeta: Glossoscolecidae) on soil fertility and bean production. Biol. Fert. Soils, 36, . 2002, pp. 313-319.

[18] Warnock D. D., Lehmann J., Kuyper T. W. etc. Mycorrhizal responses to biochar in soil concepts and mechanisms. Plant Soil, 2007, 300, pp. 9-20. 
[19] Thies J. E., Rillig M. C. Characteristics of biochar: Biological Properties, in J. Lehmann, S. Joseph (eds.): Biochar for Environmental Management-Science and Technology. Earthscan, London, 2009, pp. 85-106

[20] Chan K. Y., Xu Z. H., Lehmann J. etc. biochar: Nutrient Properties and their Enhancement, in: biochar for Environmental Management: Science and Technology. Earthscan, London \& Sterling, 2009, pp. 67-84.

[21]Erdal I., Ekinci K. Effects of vermicomposts obtained from rose oil processing wastes, dairy manure, municipal open market wastes and straw on plant growth, mineral nutrition, and nutrient uptake of corn. Journal of Plant Nutrition, 40(15), 2017, pp. 2200-2208.

[22] Erdal I., Kaya M., Kucukyumuk Z. Effects of zinc and nitrogen fertilizations on grain yield and some parameters effecting zinc bioavailability in lentil seeds. Legume Research 37 (1), 2014, pp. 55-61

[23]Zavalloni C., Alberti G., Biasiol S. etc. Microbial mineralization of biochar and wheat straw mixture in soil: A short-term study. Appl. Soil Ecol., 50, 2011, pp. 45-51.

[24] Case, S.J. McNamara, N.P. Reay, D.S. etc. The effect of biochar addition on $\mathrm{N}_{2} \mathrm{O}$ and $\mathrm{CO}_{2}$ emissions from a sandy loam soil - The role of soil aeration. Soil Biol. Biochem. 51, 2012, pp. $125-134$. 